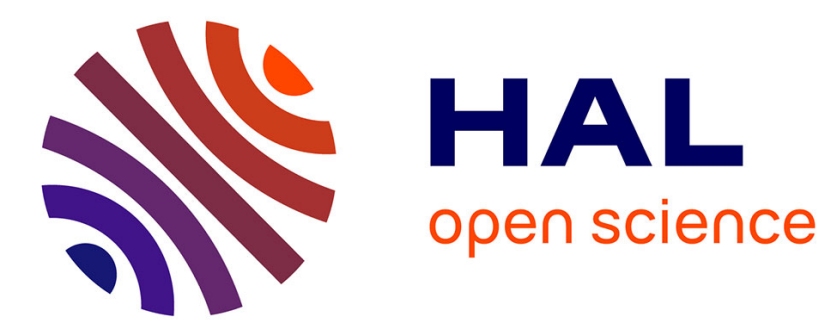

\title{
On Robustness of Phase Resetting to Cell Division under Entrainment
}

\author{
Hafiz Ahmed, Rosane Ushirobira, Denis Efimov
}

\section{To cite this version:}

Hafiz Ahmed, Rosane Ushirobira, Denis Efimov. On Robustness of Phase Resetting to Cell Division under Entrainment. Journal of Theoretical Biology, 2015, 387, pp.206-213. 10.1016/j.jtbi.2015.09.033 . hal-01211491

\author{
HAL Id: hal-01211491 \\ https://hal.inria.fr/hal-01211491
}

Submitted on 5 Oct 2015

HAL is a multi-disciplinary open access archive for the deposit and dissemination of scientific research documents, whether they are published or not. The documents may come from teaching and research institutions in France or abroad, or from public or private research centers.
L'archive ouverte pluridisciplinaire HAL, est destinée au dépôt et à la diffusion de documents scientifiques de niveau recherche, publiés ou non, émanant des établissements d'enseignement et de recherche français ou étrangers, des laboratoires publics ou privés. 


\title{
On Robustness of Phase Resetting to Cell Division under Entrainment
}

\author{
Hafiz Ahmed, Rosane Ushirobira, Denis Efimov
}

\begin{abstract}
The problem of phase synchronization for a population of genetic oscillators (circadian clocks, synthetic oscillators, etc.) is considered in this paper, taking into account a cell division process and a common entrainment input in the population. The proposed analysis approach is based on the Phase Response Curve (PRC) model of an oscillator (the first order reduced model obtained for the linearized system and inputs with infinitesimal amplitude). The occurrence of cell division introduces state resetting in the model, placing it in the class of hybrid systems. It is shown that without common entraining input in all oscillators, the cell division acts as a disturbance causing phase drift, while the presence of entrainment guarantees boundedness of synchronization phase errors in the population. The performance of the obtained solutions is demonstrated via computer experiments for two different models of circadian/genetic oscillators (Neurospora's circadian oscillation model and the repressilator).
\end{abstract}

Index Terms-Oscillation control; Phase resetting; Cell division.

\section{INTRODUCTION}

The interest in the analysis and synthesis of genetic oscillators is continuously growing these last decades [1], [2], [3], [4]. Any periodic oscillation is characterized by its frequency (or frequency spectrum), phase and amplitude. The amplitude and frequency are mainly governed by external stimulus applied to oscillators, a phenomenon called entrainment [5], [6], while the phase value is dependent on properties of the oscillator and characteristics of entrainment. This phase feature has attracted the attention of many researchers and in particular, the phase synchronization phenomenon studies are very popular [5], [6]. Phase synchronization is frequently observed in networks of oscillators, like a colony of the smallest free-living eukaryotes [7], the mammalian circadian pacemaker neural network [8], [9] or networks of neural oscillators [10], [3], [11], to mention a few. Controlled phase resetting has been studied in [12], [13], [14], [15] and for a population of oscillators in [16].

A simple but effective approach for analysis of phase resetting and dynamics for a single oscillator is based on PRC [17], [18], [5], [19]. The PRC map is calculated for the

The authors are with Non-A project @ Inria, Parc Scientifique de la Haute Borne, 40 avenue Halley, 59650 Villeneuve d'Ascq, France.

Denis Efimov is also with CRIStAL (UMR-CNRS 9189), Ecole Centrale de Lille, Avenue Paul Langevin, 59651 Villeneuve d'Ascq, France and with Department of Control Systems and Informatics, University ITMO, 49 avenue Kronverkskiy, 197101 Saint Petersburg, Russia. This work was partially supported by the Government of Russian Federation (Grant 074-U01) and the Ministry of Education and Science of Russian Federation (Project 14.Z50.31.0031).

Hafiz Ahmed is partly supported by the regional council of Nord-Pas de Calais.

Corresponding author: Hafiz Ahmed, e-mail: hafiz.ahmed@inria.fr system linearized around the limit cycle and inputs with small amplitudes. If the entraining input is a series of pulses, then a Poincaré phase map based on PRC can be calculated to predict the phase behavior [5]. Such a reduced phase model has been used in [14], [16] for pulse amplitude and timing calculation for a controlled phase resetting.

Another interesting problem that has emerged recently in [20], deals with the influence of cell division on the behavior of genetic oscillators. It has been observed that oscillations persist across cell divisions in Repressilator [21], similarly for circadian oscillations in cyanobacteria cells [22]. In [23], the persistence of circadian oscillations in culture fibroblasts under cell division has been demonstrated, and it has been noted that cell division can shift the phase in circadian cycle. A rapid phase decorrelation between daughter cells has been remarked in [24] for oscillations in the p53/Mdm2 system. Since cell division introduces a discontinuity in the oscillator dynamics (that is usually described by a system of nonlinear differential equations), then the analysis of division influence leads to the study of a hybrid or impulsive nonlinear oscillating system, which is a rather complicated problem [25], [26]. In [20], this problem has been investigated using a stochastic simulation approach, and in [27], the geometric phase approach has been adopted from quantum mechanics.

The goal of the present work is to analyze the phase behavior and synchronization under cell division in genetic oscillators using PRC formalism. A motivating example given by a simple biological model of circadian oscillations in Neurospora, is studied in Section II. The analysis of cell division influence on the phase dynamics is presented in Section III. An illustration by simulations of the obtained results is given in Section IV. General results about phase dynamics are summarized in the Appendix.

\section{Motivating EXAMPLE}

Let us consider a simple biological model of circadian oscillations in Neurospora in the following form [28]:

$$
\begin{aligned}
\dot{M}(t) & =\left(v_{s}+u(t)\right) \frac{K_{I}^{n}}{K_{I}^{n}+F_{N}^{n}(t)}-v_{m} \frac{M(t)}{K_{m}+M(t)} \\
\dot{F}_{C}(t) & =k_{s} M(t)-v_{d} \frac{F_{c}(t)}{K_{d}+F_{c}(t)}-k_{1} F_{c}(t)+k_{2} F_{n}(t) \\
\dot{F}_{N}(t) & =k_{1} F_{c}(t)-k_{2} F_{N}(t)
\end{aligned}
$$

where $M(t), F_{c}(t)$ and $F_{N}(t)$ are the concentrations (defined with respect to the total cell volume) of the frqmRNA, the cytosolic and nuclear forms of FRQ, respectively. The parameter $v_{s}$ defines the rate of $f r q$ transcription (this parameter increases in the light phase) while the influence of light (the 
external entraining input in the model (1)) is denoted by $u(t) \geq 0$. A description of the other parameters appearing in these equations can be found in [28]. The following values of parameters are proposed there: $v_{m}=0.505, v_{d}=1.4$, $k_{s}=0.5, k_{1}=0.5, k_{2}=0.6, K_{m}=0.5, K_{I}=1, K_{d}=0.13$, $n=4$ and $1 \leq v_{s}+u(t) \leq 2.5$.

For all these values, the system (1) for $u(t)=0$ has single unstable equilibrium and globally attractive limit cycle that represents a rhythmic behavior of the circadian rhythm in Neurospora with a period $T>0$. It is a continuous-time dynamical system that for any initial conditions $M(0)>0$, $F_{C}(0)>0$ and $F_{N}(0)>0$ has a continuous positive solution for all $t \geq 0$. To model the cell division in (1), it is necessary to introduce an increasing series of time instants $t_{k}>0$, $k=1,2, \ldots$ with a division at each $t_{k}$. During the division, the state variables are resetted [20], i.e. $M\left(t_{k}^{+}\right)=\lambda_{k}^{M} M\left(t_{k}\right)$, $F_{C}\left(t_{k}^{+}\right)=\lambda_{k}^{F_{C}} F_{C}\left(t_{k}\right)$ and $F_{N}\left(t_{k}^{+}\right)=\lambda_{k}^{F_{N}^{N}} F_{N}\left(t_{k}\right)$, where $M\left(t_{k}^{+}\right)$is the value of the concentration $M$ after division at instant $t_{k} ; \lambda_{k}^{M}>0, \lambda_{k}^{F_{C}}>0$ and $\lambda_{k}^{F_{N}}>0$ are parameters.

The cell division cycle can be larger than the period of oscillations $T$ [27] or similar, as in proliferating human cells [29] (where the circadian clock is a major synchronizing factor, which orchestrates daily rhythms regulating the cell division cycle), or two times faster as in cyanobacteria [30]. The values $\lambda_{k}^{M}, \lambda_{k}^{F_{C}}, \lambda_{k}^{F_{N}}$ have been selected around 0.5 in [20] (for the Goodwin model), but in [31] it has been observed in vivo that concentrations do not jump significantly after cell division. In the present work, we will accept the latter hypothesis taking $\lambda_{k}^{M}, \lambda_{k}^{F_{C}}, \lambda_{k}^{F_{N}}$ close to 1 .

The modeling of such a hybrid oscillator corresponds to a mother cell in the population, then after each division the daughter cells have a similar dynamics and forthcoming divisions augmenting the population. It is assumed that division instants $t_{k}$ for each cell are different, then the phase synchronization behavior in a population (assuming that there is no interconnection between cells) can be analyzed using (1). If the phase converges to a steady-state in this hybrid system under some conditions, then the population will be phase synchronized in some sense.

Taking the previously mentioned parameter values and $v_{s}=1.11$, the period of the autonomous oscillation of (1) is obtained as $T=19.25 \mathrm{~min}$. For these values of parameters and for the case $u(t)=0$ and $t_{k}=k T-v_{k}, k \geq 1$, where $v_{k} \in[0.15 T, 0.30 T]$ is a uniformly distributed random variable, the results of the Neurospora's circadian oscillation model simulation for the same initial conditions and different realizations of $v_{k}$ for 4 different cells undergoing divisions can be seen in Fig. 2. As we can conclude from these results the phase is diverging as it has been noted in [23], [24] and in some experiments of [20]. Next, by taking $u(t)=$ $\max \{0,0.2 \sin (\omega t)\}$ as the common external entraining input and repeating the same experiments, the results are given in Fig. 1. From Fig. 1, it is evident that the oscillations converge to a common entrained mode.

In this paper, we will try to find conditions providing both these two types of phase behavior using the PRC phase model for small inputs.

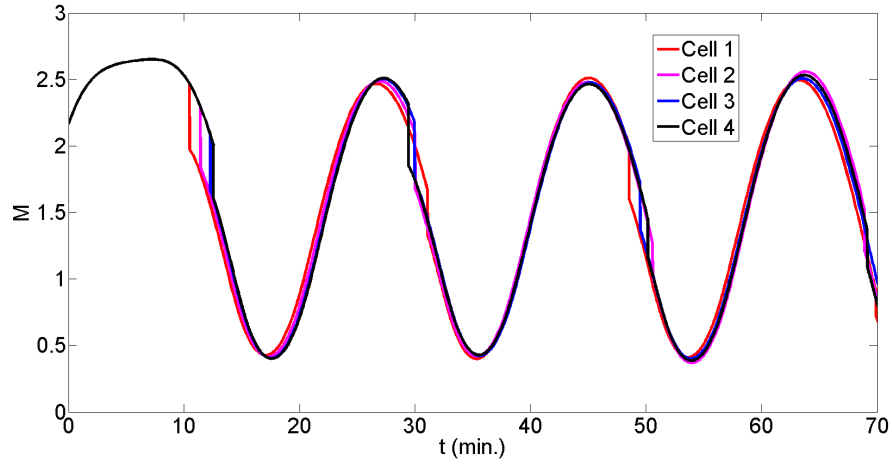

Figure 1. Oscillations of different single cells with cell divisions and common external entraining input

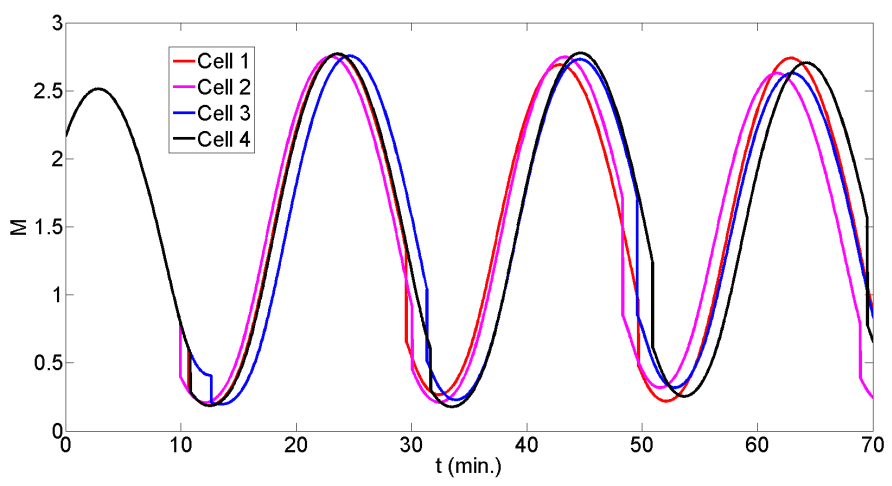

Figure 2. Oscillations of different single cells with cell divisions and without any common input

\section{PRC-BASED PHASE MODE FOR AN OSCILLATOR WITH CELL DIVISION}

This Section begins with the introduction of the formalized problem statement. Next, the reduced PRC model is introduced and the phase synchronization analysis is presented.

Details of the standard procedure for a phase model derivation for an oscillator can be found in [5], [6], [16] and they are briefly summarized in the Appendix.

\section{A. Problem statement}

Let us consider a population of $N>0$ cells (genetic oscillators) with $s=1,2, \ldots, N$ :

$$
\begin{gathered}
\dot{x}_{s}(t)=f_{s}\left(x_{s}(t), u(t)\right) \quad t \in\left[t_{s, k}, t_{s, k+1}\right), k \geq 0 \\
x_{s}\left(t_{s, k+1}^{+}\right)=\Lambda_{s, k, n} x_{s}\left(t_{s, k+1}\right) \quad k \geq 1
\end{gathered}
$$

where $x_{s}(t) \in \mathbb{R}^{n}$ is the state (concentrations of different products) of the $s^{\text {th }}$ cell and the input $u(t)$ is a periodical train of pulses

$$
u(t)=\sum_{i=0}^{+\infty} w(t-i \mathbb{T})
$$

with a pulse $w(t), w(t)=0$ for all $t \geq \mathcal{T}$ or $t \leq 0$, $\sup _{0 \leq t \leq \mathcal{T}}|w(t)| \leq W<+\infty$ and $\mathcal{T}<\mathbb{T}$ where $\mathbb{T}>0$ is the period of $u ; t_{s, 0}=0$ and $t_{s, k}, k \geq 0$ is a strictly increasing sequence of impulses (discontinuous jumps in (2)) for all $s=1,2, \ldots, N, \Lambda_{s, k, n}=\operatorname{diag}\left[\lambda_{s, k, 1}, \ldots, \lambda_{s, k, n}\right] \in \mathbb{R}^{n \times n}$ with $\lambda_{s, k, 1} \in[1-\varepsilon, 1]$ for some $\varepsilon>0$ sufficiently small. The 
periodical input $u(t)$ models the common entrainment for all cells and the discontinuities at instants $t_{s, k}, k \geq 1$ represent the cell division, the diagonal matrix $\Lambda_{s, k, n}$ determines changes in the state vector (in concentrations) after division. The instants of division $t_{s, k}$ and the concentration changes $\Lambda_{s, k, n}$ may be different for each cell.

Remark 1. Note that, formally, at each $t_{s, k}, k \geq 1$, the population should be augmented by a daughter cell, that has dynamics similar to mother one. Then, the number $N$ is continuously growing. In the present work, we will consider a fixed size of the population $N$, since as it will be shown below (and due to similarity of dynamics for newborn cells and initial conditions), the problem of phase synchronization can be analyzed using the model even for a single cell.

It is necessary to establish conditions (restrictions on $f_{s}, u$ and $t_{s, k}$ or $\Lambda_{s, k, n}$ ) under which there exists a synchronization phenomenon in the cell population (2).

\section{B. Reduced phase model under cell division}

The presence of divisions can be alternatively modeled by an additional impulsion input:

$$
\dot{x}_{s}(t)=f_{s}\left(x_{s}(t), u(t)\right)+\sum_{k=1}^{+\infty} \chi_{s, k} \delta\left(t-t_{s, k}\right),
$$

where $\delta(t)$ is a delta-impulse function, $\chi_{s, k}=$ $\left(\Lambda_{s, k, n_{s}}-I_{n_{s}}\right) x_{s}\left(t_{s, k+1}\right)$ and $I_{n_{s}}$ is the identity matrix of dimension $n_{s}, s=1,2, \ldots, N$.

Assumption 1. For each $s=1,2, \ldots, N$, the $s^{\text {th }}$ subsystem in (2), with $u=0$ and $\Lambda_{s, k, n_{s}}=I_{n_{s}}$ for all $k \geq 0$, has a limit cycle $\Gamma_{s} \subset \mathcal{A}_{s}$ with an open set of attraction $\mathcal{A}_{s} \subset \mathbb{R}^{n_{s}}$, and with period $T_{s}>0, \omega_{s}=2 \pi T_{s}^{-1}$.

This assumption says if there is no entrainment $u$ and cell division, then each cell in the population is an oscillator with the limit cycle in $\Gamma_{s}$ and period $T_{s}$. Under Assumption 1 and using the theory presented in Appendix, for each cell in (2) it is possible to define its asymptotic phase $\theta_{s} \in[0,2 \pi)$. Under additional restrictions that $\varepsilon$ and $W$ are sufficiently small, we can design a phase dynamical model of (2) in some vicinity of $\Gamma_{s}$ as in Appendix. Since the model derived in (11) is based on the first order approximation and in the system (3) there are two inputs ( $u$ and the train of impulses), by superposition principle, eq. (11) takes the form in this case for $s=1,2, \ldots, N$ :

$$
\begin{aligned}
\dot{\theta}_{s}= & \omega_{s}+Q_{s}\left(t+\theta_{s, 0} \omega_{s}^{-1}\right) b_{s}\left(t+\theta_{s, 0} \omega_{s}^{-1}\right) u(t) \\
& +Q_{s}\left(t+\theta_{s, 0} \omega_{s}^{-1}\right) \sum_{k=1}^{+\infty} \chi_{s, k} \delta\left(t-t_{s, k}\right),
\end{aligned}
$$

where

$$
b_{s}\left(t+\theta_{s, 0} \omega_{s}^{-1}\right)=\left.\frac{\partial f_{s}\left(x_{s}, u\right)}{\partial u}\right|_{x_{s}=\gamma_{s}\left(t+\theta_{s, 0} \omega_{s}^{-1}\right)}
$$

and $\gamma_{s}\left(t+\theta_{s, 0} \omega_{s}^{-1}\right)$ is a trajectory of the $s^{\text {th }}$ cell in (2) for $u=0$ and $\Lambda_{s, k, n_{s}}=I_{n_{s}}$ for all $k \geq 0$ with initial conditions in $\Gamma_{s}$ with the initial phase $\theta_{s, 0} \in[0,2 \pi], Q_{s}(t)$ is the infinitesimal PRC derived in the Appendix. This model is constructed around the base trajectory $\gamma_{s}\left(t+\theta_{s, 0} \omega_{s}^{-1}\right)$ under the assumption that the perturbed trajectory with $u \neq 0$ and $\Lambda_{s, k, n_{s}} \neq I_{n_{s}}$ stays close to that one [16]. Since such a closeness assumption is rather restrictive and may be invalid on a sufficiently long time interval (the excited trajectory can belong to a small vicinity of $\Gamma_{s}$ for sufficiently small $\varepsilon$ and $W$, but moving away from $\gamma_{s}\left(t+\theta_{s, 0} \omega_{s}^{-1}\right)$ due to a phase shift induced by external inputs), then it is better to recalculate the phase of base trajectory $\gamma_{s}\left(t+\theta_{s, 0} \omega_{s}^{-1}\right)$ after a period $\mathbb{T}$, for example (that is the idea of Poincaré phase map approach [5]). In this case, by recurrent integration of (4) (similarly as for (12)) the phase shift over the interval $[i \mathbb{T},(i+1) \mathbb{T}]$ can be evaluated as follows:

$$
\begin{gathered}
\theta_{s, i+1}=\omega_{s}\left(\mathbb{T}-T_{s}\right)+\theta_{s, i}+\mathrm{PRC}_{s}\left(\theta_{s, i}\right)+\Delta_{s, i} \\
\mathrm{PRC}_{s}(\theta)=\int_{0}^{\mathcal{T}} Q_{s}\left(t+\theta \omega_{s}^{-1}\right) b_{s}\left(t+\theta \omega_{s}^{-1}\right) w(t) d t \\
\Delta_{s, i}=\sum_{k \in \mathbb{K}_{s, i}} \chi_{s, k} Q_{s}\left(t_{s, k}+\theta_{s, i} \omega_{s}^{-1}\right)
\end{gathered}
$$

for all $s=1,2, \ldots, N$, where $\mathbb{K}_{s, i}=$ $\left\{k \geq 1 \mid t_{s, k} \in[i \mathbb{T},(i+1) \mathbb{T}]\right\} \quad$ is the set of indexes whose impulses happen in the interval $[i \mathbb{T},(i+1) \mathbb{T}]$, $\mathrm{PRC}_{s}:[0,2 \pi) \rightarrow[0,2 \pi)$ is the PRC of the $s^{\text {th }}$ oscillator for the pulse $w$ and $\Delta_{s, i} \in \mathbb{R}$ is the phase perturbation imported by cell division on the interval $[i \mathbb{T},(i+1) \mathbb{T}]$.

Remark 2. Formally, the set $\mathbb{K}_{s, i}$ can be decomposed on two parts:

$$
\begin{gathered}
\mathbb{K}_{s, i}=\mathbb{K}_{s, i}^{1} \cup \mathbb{K}_{s, i}^{2}, \\
\mathbb{K}_{s, i}^{1}=\left\{k \geq 1 \mid t_{s, k} \in[i \mathbb{T}, i \mathbb{T}+\mathcal{T}]\right\}, \\
\mathbb{K}_{s, i}^{2}=[i \mathbb{T}+\mathcal{T},(i+1) \mathbb{T}]
\end{gathered}
$$

where $\mathbb{K}_{s, i}^{1}$ characterizes the impulses arrived for $u(t) \neq 0$ and $\mathbb{K}_{s, i}^{2}$ is for $u(t)=0$ on the interval $[i \mathbb{T},(i+1) \mathbb{T}]$. Then the model (12) can be rewritten as follows:

$$
\begin{gathered}
\theta_{s, i+1}=\omega_{s}\left(\mathbb{T}-T_{s}\right)+\theta_{s, i}+\operatorname{PRC}_{s}\left(\theta_{s, i}+\Delta_{s, i}^{1}\right)+\Delta_{s, i}, \\
\Delta_{s, i}=\Delta_{s, i}^{1}+\Delta_{s, i}^{2}, \\
\Delta_{s, i}^{j}=\sum_{k \in \mathbb{K}_{s, i}^{j}} \chi_{s, k} Q_{s}\left(t_{s, k}+\theta_{s, i} \omega_{s}^{-1}\right), j=1,2 .
\end{gathered}
$$

The difference with respect to (12) is that the perturbation caused by cell division appears nonlinearly in the last model. For brevity of consideration only the case of (12) is studied below.

\section{Phase synchronization}

The model (5) for each $s=1,2, \ldots, N$ is a scalar nonlinear integrator-like discrete-time system (that is a considerable advantage with respect to (2)) with the state $\theta_{s, i}$ and external input $\omega_{s}\left(\mathbb{T}-T_{s}\right)+\Delta_{s, i}$, where the constant part represents the influence of entrainment and $\Delta_{s, i}$ is the perturbation originated by cell division.

Assume that there is no common entrainment and $u(t)=0$, then the model (5) can be simplified to a pure integrator of 
the input $\mathbb{T} \Delta_{s, i}^{-1}$, which is an average influence of pulses other interval $[i \mathbb{T},(i+1) \mathbb{T}]$ :

$$
\theta_{s, i+1}=\theta_{s, i}+\Delta_{s, i} .
$$

If $\Delta_{s, i}$ are different for each $s=1,2, \ldots, N$ and have not a zero mean, then the phase $\theta_{s, i}$ will be drifting in a unique manner for each $s=1,2, \ldots, N$. Thus, there is no phase synchronization. This is the case presented in Fig. 2 of Section II and also observed in [24], [23].

Therefore, the synchronous properties of (5) depend critically on the nonlinear function $\mathrm{PRC}_{s}$. In this work, as in [13], [14], [16], we assume that the PRC map has particular properties (it is similar to type II PRC from [32]).

Assumption 2. For all $s=1,2, \ldots, N$, the map $\mathrm{PRC}_{s}$ is continuously differentiable and there exist $0<\beta_{s} \leq 1$ and $\Theta_{s} \in[0,2 \pi)$ such that the equation $\operatorname{PRC}_{s}\left(\theta_{s}^{0}\right)=\omega_{s}\left(T_{s}-\mathbb{T}\right)$ has a solution $\theta_{s}^{0} \in[0,2 \pi)$ with

$$
-2+\beta_{s} \leq \frac{\partial \mathrm{PRC}_{s}(\theta)}{\partial \theta} \leq-\beta_{s} \quad \forall \theta \in\left[\theta_{s}^{0}-\Theta_{s}, \theta_{s}^{0}+\Theta_{s}\right] .
$$

Obviously, for $\Delta_{s, i}=0$ (no cell division), $\theta_{s}^{0}$ corresponds to a stable equilibrium of the system (5) for given $s$ with the domain of attraction $\left[\theta_{s}^{0}-\Theta_{s}, \theta_{s}^{0}+\Theta_{s}\right]$ [5].

Lemma 3. For each $s=1,2, \ldots, N$, under the Assumption 2, if $\left|\theta_{s, 0}-\theta_{s}^{0}\right| \leq \Theta_{s}-\beta_{s}^{-1} \overline{\Delta_{s}}$ where $\overline{\Delta_{s}}=\sup _{i \geq 0}\left|\Delta_{s, i}\right|<+\infty$, then

$$
\left|\theta_{s, i}-\theta_{s}^{0}\right| \leq \Theta_{s} \quad \forall i \geq 0, \lim _{i \rightarrow+\infty}\left|\theta_{s, i}-\theta_{s}^{0}\right| \leq \beta_{s}^{-1} \overline{\Delta_{s}} .
$$

Proof. Taking into account definition of $\theta_{s}^{0}$, the model (5) can be rewritten as follows:

$$
\theta_{s, i+1}=\theta_{s, i}+\operatorname{PRC}_{s}\left(\theta_{s, i}\right)-\operatorname{PRC}_{s}\left(\theta_{s}^{0}\right)+\Delta_{s, i} .
$$

Using the Mean value theorem $\left(\mathrm{PRC}_{s}\right.$ is continuously differentiable by Assumption 2), we obtain:

$$
\theta_{s, i+1}=\theta_{s, i}+\frac{\partial \mathrm{PRC}_{s}\left(\theta_{s, i}^{\prime}\right)}{\partial \theta_{s, i}^{\prime}}\left(\theta_{s, i}-\theta_{s}^{0}\right)+\Delta_{s, i},
$$

where $\theta_{s, i}^{\prime}=\mu \theta_{s, i}+(1-\mu) \theta_{s}^{0}$ for some $\mu \in[0,1]$. Define the phase error $e_{s, i}=\theta_{s, i}-\theta_{s}^{0}$, then

$$
e_{s, i+1}=\left(1+\frac{\partial \mathrm{PRC}_{s}\left(\theta_{s, i}^{\prime}\right)}{\partial \theta_{s, i}^{\prime}}\right) e_{s, i}+\Delta_{s, i}
$$

By Assumption 2, $-2+\beta_{s} \leq \frac{\partial \operatorname{PRC}_{s}\left(\theta_{s, i}^{\prime}\right)}{\partial \theta_{s, i}^{\prime}} \leq-\beta_{s}$ provided that $\left|e_{s, i}\right| \leq \Theta_{s}$. Then taking Lyapunov function $V(e)=|e|$ we have:

$$
\begin{gathered}
V\left(e_{s, i+1}\right)-V\left(e_{s, i}\right)=\left|\left(1+\frac{\partial \mathrm{PRC}_{s}\left(\theta_{s, i}^{\prime}\right)}{\partial \theta_{s, i}^{\prime}}\right) e_{s, i}+\Delta_{s, i}\right| \\
-\left|e_{s, i}\right| \\
\leq\left(\left|1+\frac{\partial \mathrm{PRC}_{s}\left(\theta_{s, i}^{\prime}\right)}{\partial \theta_{s, i}^{\prime}}\right|-1\right)\left|e_{s, i}\right|+\left|\Delta_{s, i}\right| \\
\leq-\beta_{s} V\left(e_{s, i}\right)+\left|\Delta_{s, i}\right|,
\end{gathered}
$$

that implies

$$
\left|e_{s, i}\right| \leq\left(1-\beta_{s}\right)^{i}\left|e_{s, 0}\right|+\beta_{s}^{-1} \overline{\Delta_{s}} \quad i \geq 0
$$

under assumption that $\left|e_{s, i}\right| \leq \Theta_{s}$ for all $i \geq 0$. However, if $\left|e_{s, 0}\right| \leq \Theta_{s}-\beta_{s}^{-1} \overline{\Delta_{s}}$ then $\left|e_{s, i}\right| \leq \Theta_{s}$ for all $i \geq 0$ as needed, and $\lim _{i \rightarrow+\infty}\left|e_{s, i}\right| \leq \beta_{s}^{-1} \overline{\Delta_{s}}$.

Consequently, if the influence of cell division quantified by $\overline{\Delta_{s}}$ is sufficiently small and the initial phase $\theta_{s, 0}$ lies sufficiently close to $\theta_{s}^{0}$, then the phase $\theta_{s, i}$ stays in the domain of attraction of $\theta_{s}^{0}$ and asymptotically converges to a vicinity of that equilibrium. Since all cells in population (2) yield this kind of behavior, then under these conditions, the phases are asymptotically synchronized with the error of synchronization proportional to superposition of $\max \left\{\beta_{s_{1}}^{-1} \overline{\Delta_{s_{1}}}, \beta_{s_{2}}^{-1} \overline{\Delta_{s_{2}}}\right\}$ and $\left|\theta_{s_{1}}^{0}-\theta_{s_{2}}^{0}\right|$ for any $1 \leq s_{1} \neq s_{2} \leq N$.

Theorem 4. Let assumptions 1, 2 be satisfied and $\varepsilon, W$ be sufficiently small in (2). If $\left|\theta_{s, 0}-\theta_{s}^{0}\right| \leq \Theta_{s}-\beta_{s}^{-1} \overline{\Delta_{s}}$ for all $s=1,2, \ldots, N$, then for any $1 \leq s_{1} \neq s_{2} \leq N$

$$
\begin{gathered}
\left|\theta_{s_{1}, i}-\theta_{s_{2}, i}\right| \leq\left|\theta_{s_{1}}^{0}-\theta_{s_{2}}^{0}\right|+\Theta_{s_{1}}+\Theta_{s_{2}} \quad \forall i \geq 0 \\
\lim _{i \rightarrow+\infty}\left|\theta_{s_{1}, i}-\theta_{s_{2}, i}\right| \leq\left|\theta_{s_{1}}^{0}-\theta_{s_{2}}^{0}\right|+\beta_{s_{1}}^{-1} \overline{\Delta_{s_{1}}}+\beta_{s_{2}}^{-1} \overline{\Delta_{s_{2}}} .
\end{gathered}
$$

Proof. If Assumption 1 holds and $\varepsilon, W$ are sufficiently small, then the results presented in Section V imply that a first order approximation of (2) can be used for analysis of the population behavior, and the reduced PRC model (5) can be derived for each $s=1,2, \ldots, N$. Next, since all conditions of Lemma 3 are satisfied, then the relations (6) are valid for all $s=$ $1,2, \ldots, N$. Consider the phase difference $\theta_{s_{1}, i}-\theta_{s_{2}, i}$ of any two oscillators with $1 \leq s_{1} \neq s_{2} \leq N$. Since

$$
\theta_{s_{1}, i}-\theta_{s_{2}, i}=\theta_{s_{1}, i}-\theta_{s_{1}}^{0}-\theta_{s_{2}, i}+\theta_{s_{2}}^{0}+\theta_{s_{1}}^{0}-\theta_{s_{2}}^{0}
$$

from (6):

$$
\begin{aligned}
\left|\theta_{s_{1}, i}-\theta_{s_{2}, i}\right| & \leq\left|e_{s_{1}, i}-e_{s_{2}, i}\right|+\left|\theta_{s_{1}}^{0}-\theta_{s_{2}}^{0}\right| \\
& \leq\left|\theta_{s_{1}}^{0}-\theta_{s_{2}}^{0}\right|+\Theta_{s_{1}}+\Theta_{s_{2}}
\end{aligned}
$$

and

$$
\begin{aligned}
\lim _{i \rightarrow+\infty}\left|\theta_{s_{1}, i}-\theta_{s_{2}, i}\right| & \leq \lim _{i \rightarrow+\infty}\left|e_{s_{1}, i}-e_{s_{2}, i}\right|+\left|\theta_{s_{1}}^{0}-\theta_{s_{2}}^{0}\right| \\
& \leq\left|\theta_{s_{1}}^{0}-\theta_{s_{2}}^{0}\right|+\beta_{s_{1}}^{-1} \overline{\Delta_{s_{1}}}+\beta_{s_{2}}^{-1} \overline{\Delta_{s_{2}}}
\end{aligned}
$$

as required.

This theorem establishes phase-lock behavior in the population (2), which may be composed by different cells. If all cells are identical, then the following synchronization conditions can be obtained.

Corollary 5. Let all conditions of Theorem 4 be satisfied and $\operatorname{PRC}_{s}(\theta)=\operatorname{PRC}(\theta)$ for all $s=1,2 \ldots N$ and all $\theta \in[0,2 \pi)$ (then also $\theta_{s}^{0}=\theta^{0}, \Theta_{s}=\Theta$ and $\beta_{s}=\beta$ ). If $\left|\theta_{s, 0}-\theta^{0}\right| \leq$ $\Theta-\beta^{-1} \overline{\Delta_{s}}$ for all $s=1,2 \ldots N$, then

$$
\left|\bar{\theta}_{i}-\theta^{0}\right| \leq \Theta, \quad \forall i \geq 0, \lim _{i \rightarrow+\infty}\left|\bar{\theta}_{i}-\theta^{0}\right| \leq \frac{1}{N \beta} \sum_{s=1}^{N} \overline{\Delta_{s}}
$$

where $\bar{\theta}_{i}=N^{-1} \sum_{s=1}^{N} \theta_{s, i}$ is the average phase of the population.

Proof. The result follows from Lemma 3 and Theorem 4 under assumption that all $\mathrm{PRC}_{s}$ are identical. 


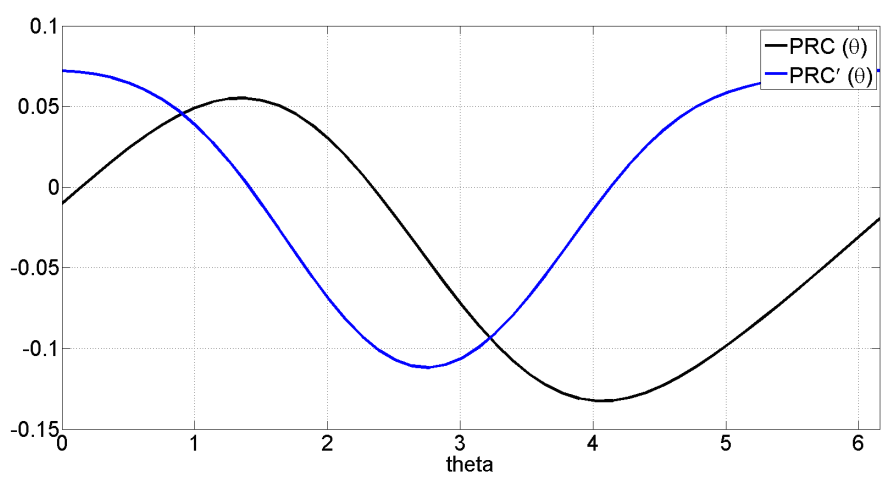

Figure 3. $\operatorname{PRC}(\theta)$ and $\operatorname{PRC}^{\prime}(\theta)$ for the input $w(t)$ for Neurospora model.

If there is no cell division, then

$$
\frac{1}{N \beta} \sum_{s=1}^{N} \overline{\Delta_{s}}=0
$$

and we recover a well-known result on phase synchronization under a periodical entrainment [5], [16].

\section{EXAMPLE}

Let us illustrate the theoretical findings obtained in the previous Section.

\section{A. Circadian oscillations in Neurospora}

Consider a population of circadian oscillators in Neurospora (1). Take all cells in the population identical with the values of parameters given in Section II. Then the Assumption 1 is satisfied for $T_{s}=T=19.25$ minutes $\left(\omega=2 \pi T^{-1}\right)$. Select

$$
w(t)= \begin{cases}\max \{0, W \sin (\omega t)\} & \text { if } 0 \leq t \leq T \\ 0 & \text { otherwise }\end{cases}
$$

with $W=0.02$. Setup the same $\Lambda_{s, k, n}$ for all $s$ and $n$ as $\Lambda_{k}$ defined by:

$$
\Lambda_{k}=\operatorname{diag}\left[\begin{array}{lll}
0.99 & 0.98 & 0.98
\end{array}\right]
$$

with $t_{k}=i T-v_{k}, k \geq 1$, where $v_{k} \in[0.15 T, 0.30 T]$ is a uniformly distributed random variable as before. The values $\varepsilon, W$ are chosen sufficiently small. For this pulse $w(t)$, the obtained $\operatorname{PRC}(\theta)$ and $\operatorname{PRC}^{\prime}(\theta)=\frac{\partial \operatorname{PRC}(\theta)}{\partial \theta}$ are shown in Fig. 3. From these plots, $\theta^{0}=2.34, \Theta=1.04$ and $\beta=0.05$, and the Assumption 2 is also satisfied. Thus, all conditions of Theorem 4 are verified.

Simulated phase behavior of (1) is shown in Fig. 4 by the blue curve (the phase value was computed by finding the closest point on the limit cycle at instants $i T$ for $i \geq 0$ and by assigning the phase of that point as $\theta_{i}$ ). The values of phase obtained by the model (12) are presented in the same figure by the red curve. As we can see, both curves are very close and that confirms all theoretical developments presented in this work, and phase asymptotically converges to a vicinity of $\theta^{0}$, then synchronization of phase would be observed for a population of circadian oscillators of Neurospora (2) as in [20] for the Godwin model.

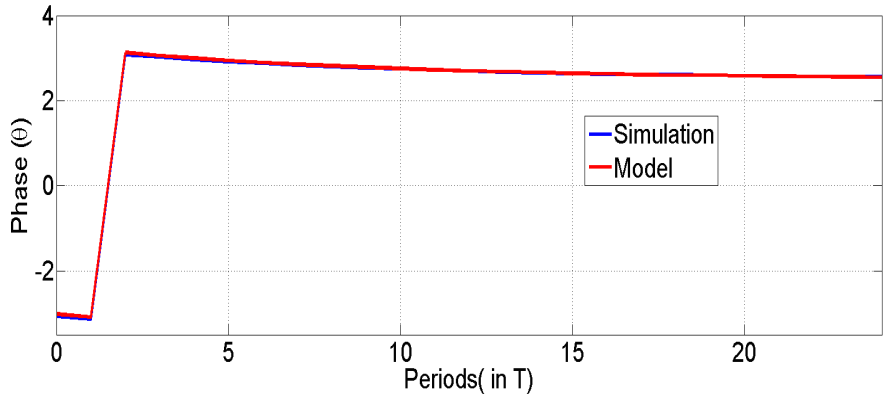

Figure 4. Phase behavior of (1)

\section{B. The Repressilator}

The repressilator [21] is a very simple genetic oscillator consisting of three genes, which can be modeled as below [20]:

$$
\begin{aligned}
& \dot{M}_{1}(t)=\alpha_{0}+\alpha_{1} \frac{K^{n}}{K^{n}+P_{3}^{n}(t)}-\delta M_{1}(t)+u(t) \\
& \dot{M}_{2}(t)=\alpha_{0}+\alpha_{1} \frac{K^{n}}{K^{n}+P_{1}^{n}(t)}-\delta M_{2}(t) \\
& \dot{M}_{3}(t)=\alpha_{0}+\alpha_{1} \frac{K^{n}}{K^{n}+P_{2}^{n}(t)}-\delta M_{3}(t) \\
& \dot{P}_{1}(t)=\beta M_{1}(t)-\gamma P_{1}(t) \\
& \dot{P}_{2}(t)=\beta M_{2}(t)-\gamma P_{2}(t) \\
& \dot{P}_{3}(t)=\beta M_{3}(t)-\gamma P_{3}(t)
\end{aligned}
$$

where the variables $M_{i}(t)$ and $P_{i}(t)$ (with $i=1,2,3$ ) represent the concentrations of mRNA and protein of the three components of the repressilator respectively and $u(t) \geq 0$ represents the external entraining input. Details about other parameters of the model can be found in [20]. We will consider the following values for (7): $\alpha_{1}=1, \alpha_{0}=0.01, K=1$, $n=2$ and $\delta=\beta=\gamma=0.1$. With these values, this model has a single equilibrium and one limit cycle and the period of autonomous oscillation is obtained as $T=116.6$ minutes.

Now, let us consider a population of identical repressilators with the previously mentioned parameters. The Assumption 1 is satisfied for $T_{s}=T=116.6 \mathrm{~min}\left(\omega=2 \pi T^{-1}\right)$. Select

$$
w(t)= \begin{cases}\max \{0, W \sin (\omega t)\} & \text { if } 0 \leq t \leq T \\ 0 & \text { otherwise }\end{cases}
$$

with $W=0.002$. As seen previously, set:

$$
\Lambda_{k}=\operatorname{diag}\left[\begin{array}{lll}
0.99 & 0.98 & 0.98
\end{array}\right]
$$

with $t_{k}=i T-v_{k}, k \geq 1$, where $v_{k} \in[0.15 T, 0.30 T]$ is a uniformly distributed random variable as before. The values $\varepsilon, W$ are chosen sufficiently small. For this pulse $w(t)$ the obtained $\operatorname{PRC}(\theta)$ and $\operatorname{PRC}^{\prime}(\theta)=\frac{\partial \operatorname{PRC}(\theta)}{\partial \theta}$ are shown in Fig. 5 , from these plots $\theta^{0}=3.9$ ( which is -2.4 in a scale between $-\pi$ to $\pi), \Theta=1.6$ and $\beta=0.01$, and Assumption 2 is also satisfied. Thus, all conditions of Theorem 4 are verified.

Simulated phase behavior of (7) is shown in Fig. 6 by the blue curve (the phase value was computed by finding the closest point on the limit cycle at instants $i T$ for $i \geq 0$ and 


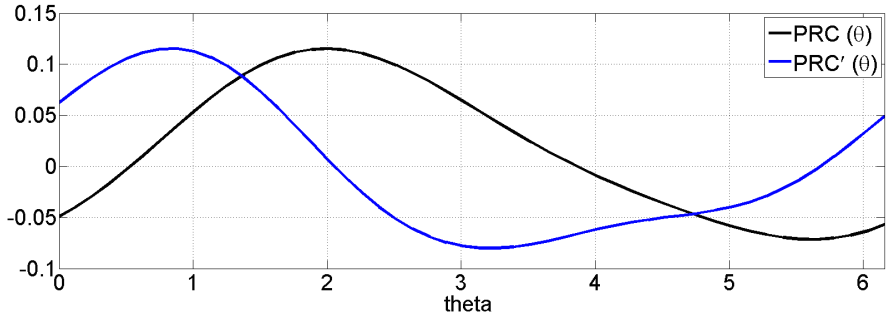

Figure 5. $\operatorname{PRC}(\theta)$ and $\operatorname{PRC}^{\prime}(\theta)$ for the input $w(t)$ for the repressilator model.

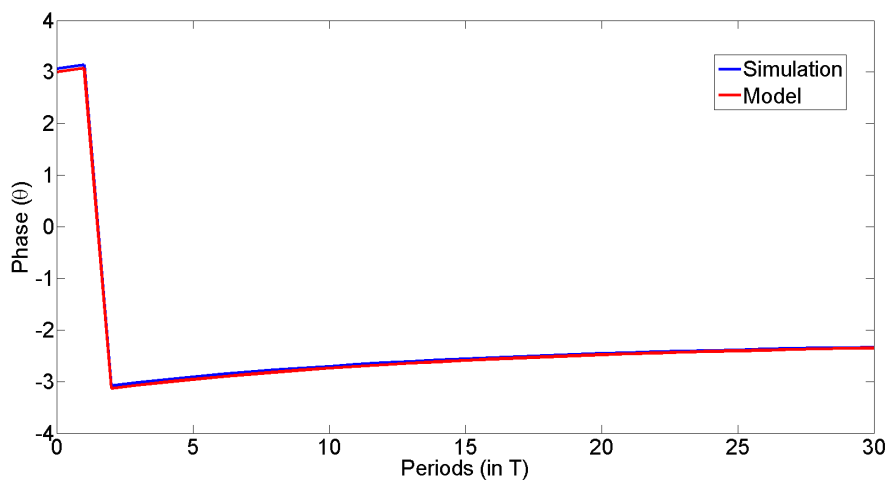

Figure 6. Phase behavior of (7)

by assigning the phase of that point as $\theta_{i}$ ). The values of phase obtained by the model (12) are presented in the same figure by the red curve. As we can see, both curves are very close and that confirms all theoretical developments presented in this work, and phase asymptotically converges to a vicinity of $\theta^{0}$, then synchronization of phase would be observed for a population of repressilator model (2) as in [20].

\section{CONClusion}

The influence of cell division on the dynamics of a population of genetic oscillators is analyzed. As it has been observed in vivo [24], [21], [22], [23], oscillations in cells are frequently quite resilient to cell division. Recently, this phenomenon has been analyzed by a stochastic simulation in [20], where phase synchronization in the population has been observed. In the present work (modeling cell division by impulses places the dynamics of population in the class of hybrid systems), analytical conditions are established of phase synchronization applying PRC model approach for small inputs. The results are illustrated by numerical experiments with two different circadian/genetic oscillator models.

\section{REFERENCES}

[1] M. Guevara, L. Glass, and A. Shrier, "Phase locking, period-doubling bifurcations, and irregular dynamics in periodically stimulated cardiac cells," Science, vol. 214, pp. 1350-1353, 1981.

[2] Y. Kuramoto, Chemical Oscillations, Waves and Turbulence. Berlin: Springer, 1984.

[3] P. Tass, Phase Resetting in Medicine and Biology. Stochastic Modeling and Data Analysis. Berlin: Springer Verlag, 1999.

[4] A. Winfree, The Geometry of Biological Time. Berlin: Springer Verlag, 1980.

[5] E. Izhikevich, Dynamical Systems in Neuroscience: The Geometry of Excitability and Bursting. The MIT press, 2007.
[6] A. Pikovsky, M. Rosenblum, and J. Kurths, Synchronization. A Universal Concept in Nonlinear Sciences. Cambridge: Cambridge University Press, 2001

[7] Q. Thommen, B. Pfeuty, P.-E. Morant, F. Corellou, F.-Y. Bouget, and M. Lefranc, "Robustness of circadian clocks to daylight fluctuations: Hints from the picoeucaryote ostreococcus tauri," PLoS Comput Biol., vol. 6, no. 11, p. e1000990, 2010.

[8] M. Antle, N. Foley, D. Foley, and R. Silver, "Gates and oscillators II: zeitgebers and the network model of the brain clock," Journal of Biological Rhythms, vol. 22, pp. 14-25, 2007.

[9] G. Zhao, "Phase organization of circadian oscillators in extended gate and oscillator models," J. Theoretical Biology, vol. 264, no. 2, pp. 367376, 2010.

[10] R. Smeal, G. Ermentrout, and J. White, "Phase-response curves and synchronized neural networks," Phil. Trans. R. Soc. B, vol. 365, pp. 24072422, 2010.

[11] C. Canavier and S. Achuthan, "Pulse coupled oscillators and the phase resetting curve," Math Biosci., vol. 226, no. 2, pp. 77-96, 2010.

[12] N. Bagheri, J. Stelling, and F. Doyle, "Circadian phase entrainment via nonlinear model predictive control," Int. J. Robust and Nonlinear Control, vol. 17, pp. 1555-1571, 2007

[13] P. Danzl and J. Moehlis, "Spike timing control of oscillatory neuron models using impulsive and quasi-impulsive charge-balanced inputs," in Proc. 29th American Control Conference (ACC2008), (Seattle, USA), pp. 171-176, 2008.

[14] D. Efimov, P. Sacre, and R. Sepulchre, "Controlling the phase of an oscillator: a phase response curve approach," in Proc. IEEE CDC 2009, pp. 7692-7697, 2009.

[15] D. Efimov, "Phase resetting control based on direct phase response curve," Journal of Mathematical Biology, vol. 63, no. 5, pp. 855-879, 2011.

[16] D. Efimov, "Phase resetting for a network of oscillators via phase response curve approach," Biological Cybernetics, vol. 109, no. 1, pp. 95-108, 2015.

[17] L. Glass, Y. Nagai, K. Hall, M. Talajic, and S. Nattel, "Predicting the entrainment of reentrant cardiac waves using phase resetting curves," Physical Rev. E, vol. 65, pp. 65-74, 2002.

[18] W. Govaerts and B. Sautois, "Computation of the phase response curve: A direct numerical approach," Neural Computation, vol. 18, pp. 817847, 2006.

[19] P. Sacré and R. Sepulchre, "Sensitivity analysis of oscillator models in the space of phase-response curves: oscillators as open systems," Control Systems, IEEE, vol. 34, no. 2, pp. 50-74, 2014.

[20] D. Gonze, "Modeling the effect of cell division on genetic oscillators," Journal of Theoretical Biology, vol. 325, no. 0, pp. 22-33, 2013.

[21] M. Elowitz and S. Leibler, "A synthetic oscillatory network of transcriptional regulators," Nature, vol. 403, pp. 335-338, 2000.

[22] I. Mihalcescu, W. Hsing, and S. Leibler, "Resilient circadian oscillator revealed in individual cyanobacteria," Nature, vol. 430, pp. 81-85, 2004.

[23] E. Nagoshi, C. Saini, C. Bauer, T. Laroche, F. Naef, and U. Schibler, "Circadian gene expression in individual fibroblasts: Cell-autonomous and self-sustained oscillators pass time to daughter cells," Cell, vol. 119 , no. 5, pp. 693-705, 2004.

[24] N. Geva-Zatorsky, N. Rosenfeld, S. Itzkovitz, R. Milo, A. Sigal, E. Dekel, T. Yarnitzky, Y. Liron, P. Polak, G. Lahav, and U. Alon, "Oscillations and variability in the p53 system," Mol. Syst. Biol., vol. 2, p. 0033, 2006.

[25] A. Churilov, A. Medvedev, and P. Mattsson, "Periodical solutions in a pulse-modulated model of endocrine regulation with time-delay," IEEE Transactions on Automatic Control, vol. 59, no. 3, pp. 728-733, 2014.

[26] D. Efimov, W. Perruquetti, and A. Shiriaev, "On existence of oscillations in hybrid systems," Nonlinear Analysis: Hybrid Systems, vol. 12, no. 5, pp. 104-116, 2014.

[27] D. S. Tourigny, "Geometric phase shifts in biological oscillators," Journal of Theoretical Biology, vol. 355, no. 0, pp. 239-242, 2014.

[28] J.-C. Leloup, D. Gonze, and A. Goldbeter, "Limit cycle models for circadian rhythms based on transcriptional regulation in drosophila and neurospora," J. Biological Rhythms, vol. 14, pp. 433-448, 1999.

[29] S. Bernard and H. Herzel, "Why do cells cycle with a 24 hour period?," Genome Inform., vol. 17, pp. 72-79, 2006.

[30] T. Mori, B. Binder, and C. Johnson, "Circadian gating of cell division in cyanobacteria growing with average doubling times of less than 24 hours," Proc. Natl. Acad. Sci. U.S.A., vol. 93, pp. 10183-10188, 1996.

[31] N. Cookson, S. Cookson, L. Tsimring, and J. Hasty, "Cell cycledependent variations in protein concentration," Nucleic Acids Research, vol. 38 , no. 8 , pp. 2676-2681, 2010. 
[32] D. Hansel, G. Mato, and C. Meunier, "Synchrony in excitatory neural networks," Neural Comput., vol. 7, pp. 307-337, 1995.

[33] A. Andronov, A. Vitt, and A. Khaikin, Theory of oscillators. Dover, reprint ed., 1987.

[34] V. Yakubovich and V. Starzhinskii, Linear differential equations with periodic coefficients. Wiley, 1975.

APPENDIX: PhASE MODEL IN VICINITY OF A LIMIT CYCLE

\section{A. Linearized model}

Consider a (smooth) dynamical system

$$
\dot{x}=f(x, u), \quad x \in \mathbb{R}^{n}, \quad u \in[-U, U] \subset \mathbb{R}, U>0 .
$$

Denote by $x\left(t, x_{0}, u\right)$ a solution of (8) with the initial condition $x_{0}$ and input $u$ and assume that for $u(t) \equiv 0, t \geq 0$ and some $x_{0} \in \mathbb{R}^{n}$ the system (8) has (non-constant) $T$-periodic solution $x\left(t, x_{0}, 0\right)=\gamma(t)=\gamma(t+T) \in \mathbb{R}^{n}, t \geq 0$. Then the corresponding limit cycle, described by the set $\Gamma=\left\{x \in \mathbb{R}^{n} \mid\right.$ $x=\gamma(t), 0 \leq t<T\}$, attracts a non-empty open bounded set of initial conditions $\mathcal{A} \subset \mathbb{R}^{n}, \Gamma \subset \mathcal{A}$, and the linearized system

$$
\begin{gathered}
\delta \dot{x}(t)=A(t) \delta x(t)+b(t) u(t)+d(\delta x(t), \gamma(t), u(t)), \\
A(t)=\left.\frac{\partial f(x, u)}{\partial x}\right|_{x=\gamma(t)}, \quad b(t)=\left.\frac{\partial f(x, u)}{\partial u}\right|_{x=\gamma(t)}
\end{gathered}
$$

has $n-1$ multipliers strictly inside the unit cycle and one multiplier equals to 1 [33], [34], where $\delta x(t)=x(t)-\gamma(t)$, the matrix function $A$ and the vector function $b$ are $T$-periodic due to properties of $\gamma$; the function $d(\delta x(t), \gamma(t), u(t))$ represents the higher order terms with respect to $\delta x(t)$ in the system (8) linearization and for all $x \in \mathcal{A}$ and $|u| \leq U$ there exist $d_{1}>0$, $d_{2}>0$ such that (the function $d$ contains products of $\delta x$ and $u$ with power 2 and higher):

$$
|d(\delta x, \gamma, u)| \leq d_{1}|\delta x|^{2}+d_{2} u^{2} .
$$

Multipliers are the eigenvalues of the monodromy matrix $M=$ $\Phi(T)$ defined via the fundamental matrix function $\Phi$ of the system (9) and the solution of adjoint system $\Psi$ :

$$
\dot{\Phi}(t)=A(t) \Phi(t), \Phi(0)=I ; \dot{\Psi}(t)=-A(t)^{T} \Psi(t), \Psi(0)=I,
$$

where $I$ is the identity matrix and $\Phi(t)^{T} \Psi(t)=I$.

\section{B. Phase variables}

Any point $x_{0} \in \Gamma$ can be characterized by a scalar phase $\varphi_{0} \in[0,2 \pi)$, that uniquely determines the position of the point $x_{0}$ on the limit cycle $\Gamma$ ( $\Gamma$ is a one-dimensional closed curve in $\mathbb{R}^{n}$ ) [5], [6]. The smooth bijective phase map $\vartheta: \Gamma \rightarrow[0,2 \pi)$ assigns to each point $x_{0} \in \Gamma$ the corresponding phase $\varphi_{0}=$ $\vartheta\left(x_{0}\right)$. Any solution of the system (8) $x\left(t, x_{0}, 0\right)$ with $x_{0} \in \Gamma$ satisfies $x\left(t, x_{0}, 0\right)=\gamma\left(t+\varphi_{0} \omega^{-1}\right)$, where $\omega=2 \pi T^{-1}$ is the system frequency, provided we choose the convention $\gamma(t)=$ $x\left(t, \vartheta^{-1}(0), 0\right)$, then we can define $\vartheta^{-1}(\varphi)=\gamma\left(\varphi \omega^{-1}\right)$. The phase variable $\varphi: \mathbb{R}_{+} \rightarrow[0,2 \pi)$ is defined for the trajectories $x\left(t, x_{0}, 0\right), x_{0} \in \Gamma$ as $\varphi(t)=\vartheta\left(x\left(t, x_{0}, 0\right)\right)=$ $\vartheta\left(\gamma\left(t+\varphi_{0} \omega^{-1}\right)\right)$. Due to the periodic nature of $\gamma(t)$, the function $\varphi(t)$ is also periodic. Moreover the function $\vartheta$ can be defined providing $\varphi(t)=\omega t+\varphi_{0}$ and $\dot{\varphi}(t)=\omega$ [5], [6].
The notion of phase can be extended to any solution $x\left(t, x_{0}, 0\right)$ starting in the attraction set $\mathcal{A}$ of the limit cycle. By definition, for all $x_{0} \in \mathcal{A}$ there exists an asymptotic phase $\theta_{0} \in[0,2 \pi)$ such that

$$
\lim _{t \rightarrow+\infty}\left|x\left(t, x_{0}, 0\right)-\gamma\left(t+\theta_{0} \omega^{-1}\right)\right|=0 .
$$

Then there exists the asymptotic phase map $v: \mathcal{A} \rightarrow[0,2 \pi)$ connecting a point $x_{0} \in \mathcal{A}$ and the corresponding phase $\theta_{0}$, i.e. $\theta_{0}=v\left(x_{0}\right)$ and by construction $v\left(x_{0}\right)=\vartheta\left(x_{0}\right)$ for all $x_{0} \in \Gamma$. The asymptotic phase variable $\theta: \mathbb{R}_{+} \rightarrow[0,2 \pi)$ is derived as $\theta(t)=v\left(x\left(t, x_{0}, 0\right)\right), t \geq 0$. In the case $\varphi(t)=\omega t+\varphi_{0}$ we have $\theta(t)=\omega t+\theta_{0}$ and $\dot{\theta}(t)=\omega$, which implies time invariance of this map: if $v\left(x_{1}\right)=v\left(x_{2}\right)$, then $v\left(x\left(t, x_{1}, 0\right)\right)=v\left(x\left(t, x_{2}, 0\right)\right)$ for all $t \geq 0$ and $x_{1}, x_{2} \in \mathcal{A}$ [5]. The initial conditions $x_{1}, x_{2} \in \mathcal{A}$ having the same asymptotic phase determine the isochrone curves [5].

The notion of asymptotic phase variable can be extended to a generic $u(t) \neq 0, t \geq 0$ provided that the corresponding trajectory $x\left(t, x_{0}, u\right)$ stays in the set $\mathcal{A}$ for all $t \geq 0$. In this case the asymptotic phase variable can be defined in a trivial way as $\theta(t)=v\left(x\left(t, x_{0}, u\right)\right), t \geq 0$. Then the variable $\theta\left(t^{\prime}\right)$ at an instant $t^{\prime} \geq 0$ evaluates the asymptotic phase of the point $x\left(t^{\prime}, x_{0}, u\right)$ if one would pose $u(t)=0$ for $t \geq t^{\prime}$. Dynamics of the asymptotic phase variable $\theta(t)$ in the generic case for $u(t) \neq 0, t \geq 0$ is hard to derive. A local model obtained in a small neighborhood of the limit cycle for infinitesimal inputs is presented below [5], [16].

\section{Infinitesimal PRC}

Consider the case $u(t)=0$ for $t \geq 0$, then by definition $\dot{\gamma}(t)=f(\gamma(t), 0), \ddot{\gamma}(t)=A(t) \dot{\gamma}(t)$ and $\dot{\gamma}(t)=\Phi(t) \dot{\gamma}(0)$ for all $t \geq 0$. Therefore, $\dot{\gamma}(0)=f(\gamma(0), 0)$ is the left eigenvector of the matrix $M$ for the eigenvalue $\lambda_{1}(M)=1$. There exists a right eigenvector $m \in \mathbb{R}^{n}$ such that $m^{T} M=m^{T}$ and $m^{T} \dot{\gamma}(0)=\omega$. Finally, define $Q(t)=m^{T} \Psi(t)^{T}$ then

$$
\begin{aligned}
Q(t) f(\gamma(t), 0) & =m^{T} \Psi(t)^{T} f(\gamma(t), 0) \\
& =m^{T} \Psi(t)^{T} \Phi(t) \dot{\gamma}(0)=m^{T} \dot{\gamma}(0)=\omega
\end{aligned}
$$

From another side, $\theta(t)=v(\gamma(t))=\omega t+\theta(0)$ and

$$
\omega=\dot{\theta}=\left.\frac{\partial v(x)}{\partial x}\right|_{x=\gamma(t)} f(\gamma(t), 0) .
$$

Therefore $Q(t)=\left.\frac{\partial v(x)}{\partial x}\right|_{x=\gamma(t)}+\zeta(t)$, where $\zeta(t)$ is a rowvector orthogonal to $f(\gamma(t), 0)$. Since $m$ is the eigenvector corresponding to $\lambda_{1}(M)=1$ (or movement on the limit cycle), then $Q(t)=m^{T} \Psi(t)^{T}$ is independent of perturbations orthogonal to the limit cycle flow $f(\gamma(t), 0)$ and the convention

$$
Q(t)=\left.\frac{\partial v(x)}{\partial x}\right|_{x=\gamma(t)}=m^{T} \Psi(t)^{T}
$$

is adopted. The first equality in (10) explains the physical meaning of $Q(t)$, while the last equality in (10) is used for numerical calculation. The function $Q(t)$ is $T$-periodic by construction.

The function $Q\left(\phi \omega^{-1}\right)$ for phase $\varphi \in[0,2 \pi)$ is called infinitesimal PRC [5], it serves as the phase response characteristics for a delta-impulse input. 


\section{Phase dynamics}

Consider the case $u(t) \neq 0$ (we assume that $x\left(t, x_{0}, u\right) \in \mathcal{A}$ for all $t \geq 0$ ), then

$$
\begin{aligned}
\dot{\theta}(x(t))= & \dot{\theta}(\gamma(t)+\delta x(t)) \\
= & \left.\frac{\partial v(x)}{\partial x}\right|_{x=\gamma(t)+\delta x(t)} f(\gamma(t)+\delta x(t), u(t)) \\
= & \left.\frac{\partial v(x)}{\partial x}\right|_{x=\gamma(t)} f(\gamma(t), u(t)) \\
& +r_{1}(\gamma(t), \delta x(t), u(t))^{T} \delta x(t),
\end{aligned}
$$

where the term $r_{1}(\gamma(t), \delta x(t), u(t))^{T} \delta x(t)$ corresponds to the powers of $\delta x(t)$ higher than one in the Taylor series of the function $\dot{\theta}(\gamma(t)+\delta x(t))$ with respect to the variable $\delta x(t)$. From above, the quantity $\dot{\theta}(t)=\omega$ should be satisfied for $u(t)=0$, therefore $\left.\frac{\partial v(x)}{\partial x}\right|_{x=\gamma(t)} f(\gamma(t), 0)+$ $r_{1}(\gamma(t), \delta x(t), 0)^{T} \delta x(t)=\omega$, which implies the property $r_{1}(\gamma(t), \delta x(t), 0)=0$. Next,

$$
\begin{aligned}
\dot{\theta}(x(t))= & \left.\frac{\partial v(x)}{\partial x}\right|_{x=\gamma(t)} f(\gamma(t), 0) \\
& +\left.\left.\frac{\partial v(x)}{\partial x}\right|_{x=\gamma(t)} \frac{\partial f(\gamma(t), u)}{\partial u}\right|_{u=0} u(t) \\
& +g(\gamma(t), \delta x(t), u(t)), \\
g(\gamma, \delta x, u)= & r_{1}(\gamma, \delta x, u)^{T} \delta x+r_{2}(\gamma, u) u^{2},
\end{aligned}
$$

where $r_{2}(\gamma, u) u^{2}$ represents the terms with powers two and higher for the Taylor series of the function $\left.\frac{\partial v(x)}{\partial x}\right|_{x=\gamma(t)} f(\gamma(t), u(t))$ with respect to the control $u$. For all $x \in \mathcal{A}$ and $|u| \leq U$ there are $g_{1}>0$ and $g_{2}>0$ such that $|g(\gamma, \delta x, u)| \leq g_{1} u^{2}+g_{2}|\delta x|^{2}$. Recalling the previously introduced designations, we obtain

$$
\dot{\theta}=\omega+Q(t) b(t) u(t)+g(\gamma(t), \delta x(t), u(t)) .
$$

This model has been derived around the solution $\gamma(t)$, due to the periodicity of the solution $\gamma\left(t+\phi \omega^{-1}\right), \phi \in[0,2 \pi)$ and $u$, the model for $\gamma\left(t+\phi \omega^{-1}\right)$ has a similar form [5], [16]:

$$
\begin{aligned}
\dot{\theta}= & \omega+Q\left(t+\phi \omega^{-1}\right) b\left(t+\phi \omega^{-1}\right) u(t) \\
& +g\left(\gamma\left(t+\phi \omega^{-1}\right), \delta x(t), u(t)\right) .
\end{aligned}
$$

Skipping the residual function $g$ we obtain the first order approximation of the phase model:

$$
\dot{\theta}=\omega+Q\left(t+\phi \omega^{-1}\right) b\left(t+\phi \omega^{-1}\right) u(t) .
$$

Since the property $|g(\gamma, \delta x, u)| \leq g_{1} u^{2}+g_{2}|\delta x|^{2}$ holds for all $x \in \mathcal{A}$ and $|u| \leq U$, such an approximation is rather accurate for a sufficiently small $U$.

Assume that the input $u(t)=w(t)$, where $w(t)$ has a pulselike form, i.e. $|w(t)| \leq U$ for all $0<t<\mathcal{T}<T$ and $w(t)=0$ for all $t \geq \mathcal{T}$ or $t \leq 0$. Then integration of (11) yields for

$$
t \geq \mathcal{T}:
$$

$$
\begin{aligned}
\theta(t)= & \omega t+\theta(0) \\
& +\int_{0}^{t} Q\left(\tau+\theta(0) \omega^{-1}\right) b\left(\tau+\theta(0) \omega^{-1}\right) u(\tau) d \tau \\
= & \omega t+\theta(0)+\operatorname{PRC}[\theta(0)], \\
\operatorname{PRC}(\theta)= & \int_{0}^{\mathcal{T}} Q\left(\tau+\theta \omega^{-1}\right) b\left(\tau+\theta \omega^{-1}\right) u(\tau) d \tau .
\end{aligned}
$$

The map $\operatorname{PRC}(\theta), \theta \in[0,2 \pi)$ is defined for the particular pulse $w$ (by definition $-\pi \leq \operatorname{PRC}(\theta)<\pi$ for all $\theta \in[0,2 \pi)$ ), it tabulates the phase shift by the pulse $w$ [5], [6]. For the $w(t)=\delta(t-\eta)$ with $\eta \in(0, \mathcal{T})$, where $\delta(t)$ is impulse input we obtain the infinitesimal PRC

$$
\operatorname{iPRC}(\theta)=Q\left(\eta+\theta \omega^{-1}\right) b\left(\eta+\theta \omega^{-1}\right),
$$

which defines the phase shift under an impulse input. 